\title{
An X-Y table control system using the dual loop PLL motion controller
}

\author{
Hidekazu Machida $^{\mathrm{a}^{*}}$, Michinobu Kambara ${ }^{\mathrm{b}}$, Takihara Hirotaka ${ }^{\mathrm{c}}$ \\ a National Institute of Technology, Maizuru College, Kyoto 625-8511, Japan \\ ${ }^{\mathrm{b}}$ Factory-Automation Electronics Inc., Osaka 533-0033, Japan \\ ${ }^{\mathrm{c}}$ ROHM Co., Ltd., Kyoto 615-8585, Japan \\ machida@maizuru-ct.ac.jp
}

\begin{abstract}
PLL is used for many electronic equipments, and effective in motion control as well, to provide high level of speed and position accuracy. A problem with PLL control is "cycle slip" in cases with rapidly changing speed, leading to large phase error to cause harmful vibrations. We propose prevention method for this problem, using "multi-state" phase detector is proposed. Larger input range than usual 3-state detector can absorb transient slips. Its effectiveness is confirmed with simulation and experimental results. In this article, We try to apply this system to $\mathrm{X}-\mathrm{Y}$ table including bi-direction acceleration.
\end{abstract}

Keywords: dual-loop PLL, cycle slip , motion control

\section{Introduction}

PLL, or Phase-Locked Loop ${ }^{(1)}$, is characteristic in that its input and output frequencies are completely the same, by maintaining the phase difference between in and out to zero or very small by "phase synchronization".

However, when it is utilized in motion control system ${ }^{(2)}$, there can be large phase error due to too slow responses, if input includes acceleration. This error may result in large phase deviation called "cycle slip" or "pull-in" process to recover synchronization, leading to harmful vibrations.

Traditionally ${ }^{(3)}$, the PLL motor control including acceleration, detecting the slipped pulse and interpolating that in input as shown in appendix, in this method, the precision couldn't be secured.

In order to cope with these large acceleration, there is a dual-loop PLL control ${ }^{(4)}$, a feed-forward, 2-DOF control system. However, even in this PLL, if input includes rapid acceleration, there can be a cycle slip to occur .
These problems can be remedied by introducing multi-state phase detector to detect phase-lockout, then reinforcing feed-forward gain. Its effectiveness is confirmed with simulation and experimental results ${ }^{(5)}$.

In this article, We try to apply this system to X-Y table including bi direction acceleration.

\section{PLL system}

\subsection{Basic PLL system}

Block diagram of basic PLL ${ }^{(1)}$ is shown in Fig.1. It consists of Phase Detector, PD for short, Loop Filter, LF and voltage-controlled oscillator, VCO. When this feedback system is stable in operation, it is called locked, and phase error is zero or very small, leading to synchronization between the input and output frequencies.

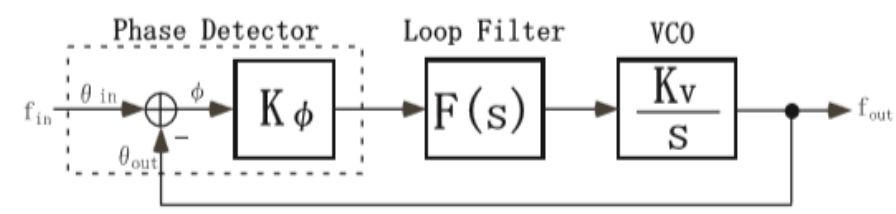

Fig. 1. basic PLL construction

When Loop filter, is PI-type, as $F(s)=\left(\tau_{2} s+1\right) / \tau_{1} s$, transfer function $H(s)$, from input phase $\theta$ in to feedback phase $\theta$ out, can be obtained as follows,

$$
H(s)=\frac{\theta_{\text {out }}(s)}{\theta_{\text {in }}(s)}=\frac{K \tau_{2} s+K}{\tau_{1} s^{2}+K \tau_{2} s+K}
$$

where $K=K_{\phi} K_{V}$. Steady-state phase error can be analyzed from the following final value theorem:

$$
\lim _{t \rightarrow \infty} \phi(t)=\lim _{s \rightarrow 0} s[1-H(s)] \theta_{i n}(s)
$$


When input phase signal $\theta_{i n}(s)$ has three components: phase step $\Delta \theta$, frequency step $\Delta \omega$, and frequency ramp R.

$$
\theta_{i n}(s)=\frac{R}{s^{3}}+\frac{\Delta \omega}{s^{2}}+\frac{\Delta \theta}{s}
$$

Steady-state phase error is zero for $\Delta \theta$ and $\Delta \omega$, but not zero for $R$, because:

$$
\lim _{t \rightarrow \infty} \phi(t)=\frac{\tau_{1}}{K} R
$$

\subsection{Dual-loop PLL system}

Dual-loop PLL ${ }^{(3)}$ is intended to be used in fast-moving terminals such as satellites, to solve the problem of Doppler effect. In the dual-loop PLL shown in Fig. 2, the first loop does not have LF, but the second loop has a filter that maintains phase margin. VCO of the second PLL is driven both by the LF output of the second loop and by the PD output of the first loop. Dual-loop PLL output is VCO output of the second loop.

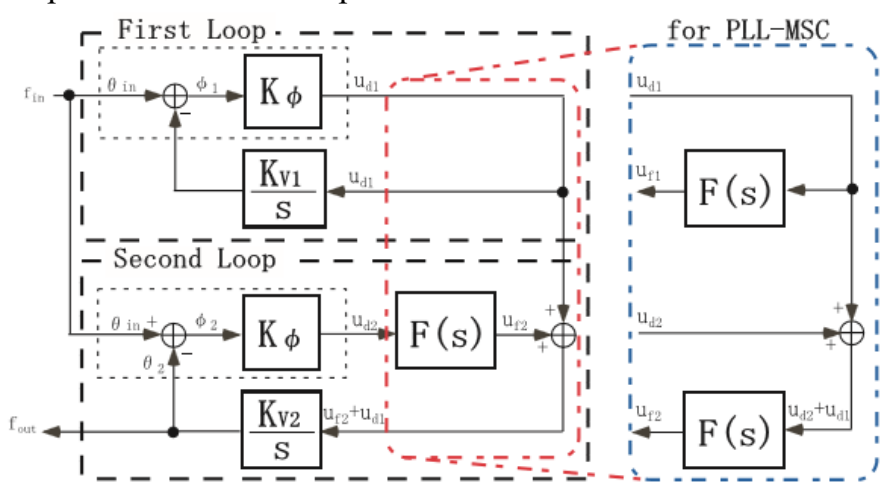

Fig. 2. Dual-loop PLL construction

When input signal has the form of Eq. (4), steady phase error can be zero for frequency ramps with constant acceleration $R$ in the input. It is for cases where $K_{V 1}$ and $K_{V 2}$ which are VCO gain of first and second loop, are equal,

$$
\lim _{t \rightarrow \infty} \phi(t)=\frac{\tau_{1}\left(K_{V 1}-K_{V 2}\right)}{K_{V 1} K_{V 2}} R=0
$$

That is, it can reject phase error for input acceleration and track to rapidly change input, because the system forms a feed-forward structure in the 2-DOF control systems.

\section{Dual-loop PLL-MSC system}

\subsection{Basic PLL-MSC system}

PLL-MSC system $^{(2)}$ can be implemented only by replacing VCOs transfer function $K_{V}(s)$ in Fig. 1 by motor + rotary encoders transfer function $K_{V}(s)=K_{m} /\left(s\left(T_{m} s+1\right)\right)$.

Because this PLL-MSC system is still only of type two, from the internal model principle, phase error for input acceleration remains as:

$$
\lim _{t \rightarrow \infty} \phi(t)=\frac{T_{m} \tau_{1}}{K} R
$$

where $K=K_{\phi} K_{m}$.

\subsection{Dual-loop PLL-MSC system}

However, to apply the dual-PLL system to MSC system, there is a problem of delay, because MSC differs from circuit. Here, simple replacement of VCO in the dual-loop PLL by a motor with encoder (Fig.2) is not enough for constructing a control system. It is because the motor reverses rotation for negative input from $\mathrm{PD}$.

Then, we moved LF to the feedback loop of both the first and second loops as shown in the right side of Fig.2.

When input signal has the form of Eq.(3), steady phase error can be reduced to zero for frequency ramps with constant acceleration $R$ in the input. It is for cases where VCO gain $K_{V}$ and motor gain $K_{m}$ are equal, because:

$$
\lim _{t \rightarrow \infty} \phi(t)=\frac{\tau_{1}\left(K_{V}-K_{m}\right)}{K_{\phi} K_{V} K_{m}} R=0
$$

\subsection{Simulation}

MATLAB simulations for single- and dual- loop PLL-MSCs are shown in Fig.3(a) and (b), respectively. Here, squared ramp corresponds to constant acceleration. Steady-state phase is not coincided to inputs in (a), but is coincided inputs in (b).
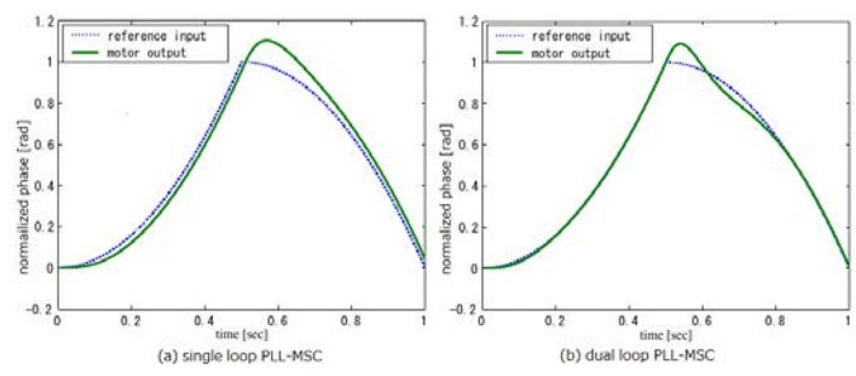

Fig. 3. Responses for inputs with constant acceleration by MATLAB

Thus, dual-loop PLL MSC features better steady phase error and rapid responses, by feed-forwarding through a low-order controller. 


\subsection{Experimental result}

We implement dual-loop PLL-MSC by using the FPGA. Fig.4 show, constant acceleration responses are observed using $\mathrm{F} / \mathrm{V}$-converter for motor encoder output.
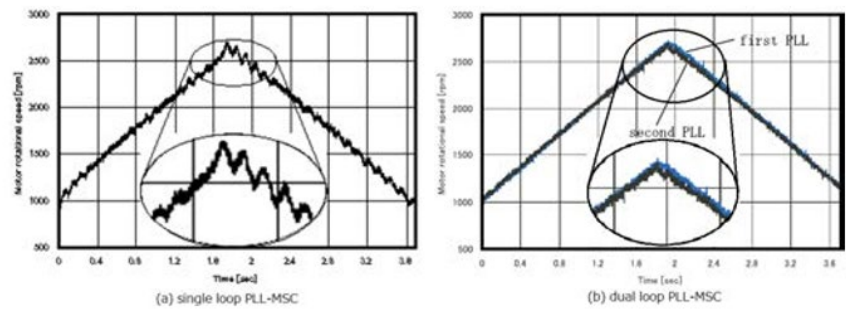

Fig. 4. accelerated speed response

As a whole, Fig.4 shows waveforms similar to Fig.3, However, one phenomenon to be noted in (a) is vibration. It is not the one shown in Fig.4 (b) and is caused by PLL cycle slips, frequently occurring in the single loop. On the other hand in (b), dual-loop PLL MSC, there is almost no vibration and phase error, but with some transient overshoot on the top.

\section{4. reduction of cycle slips}

As described so far, dual-loop PLL can eliminate some critical problems of usual PLL MSC. However, it still has one drawback, especially when input changes quickly or motor load is heavy: that is "cycle slip".

The cause "cycle slip" is when input has acceleration is too quick, phase error becomes too large and exceeds the linear range of phase comparator. The occasion of the extreme is when input acceleration include discontinuity, transient overshoot is resulted and linear range is exceeded.

\subsection{Principle of extending linear range for PD}

When acceleration of input is too large, phase error becomes excessive and exceeds the linear range of phase comparator. Therefore, the first consideration is to expand the range of the phase comparator.

Usually phase comparator for the PLL-MSC is 3-state PFD(Phase Frequency Detector) is shown in Fig.5

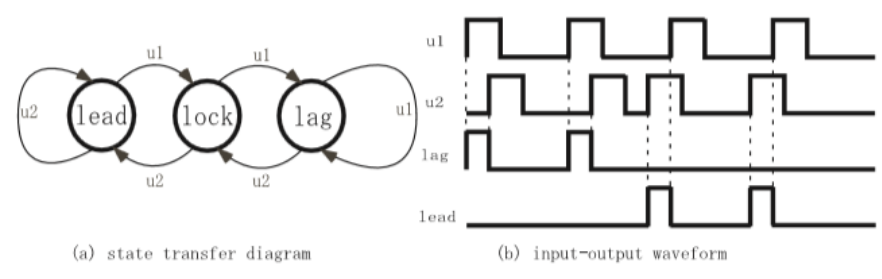

Fig. 5. PFD : Phase Frequency Detector range
Fig. 6 is an example. It is the case of accommodating up to $\pm 6 \pi=1080$ [degree] with 7 internal states.

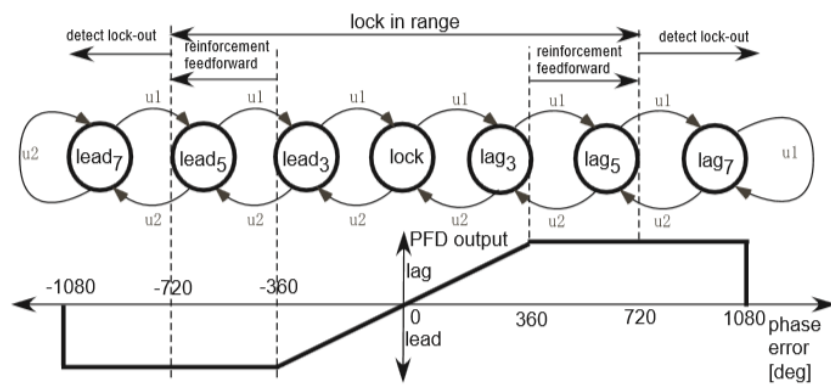

Fig. 6. multi-state PFD ranges

Then, we propose that, when state going out from 3- to 5-state, feed-forward gain is increased, to prevent the state further going into 7-state.

In this method, the feed forward amount reinforcement is not performed for small phase errors. Moreover, input speed is changed from acceleration to constant, then returning to the conventional (one-degree of freedom) PLL motor speed control system. Fig.7 shows that block diagram of the above control method.

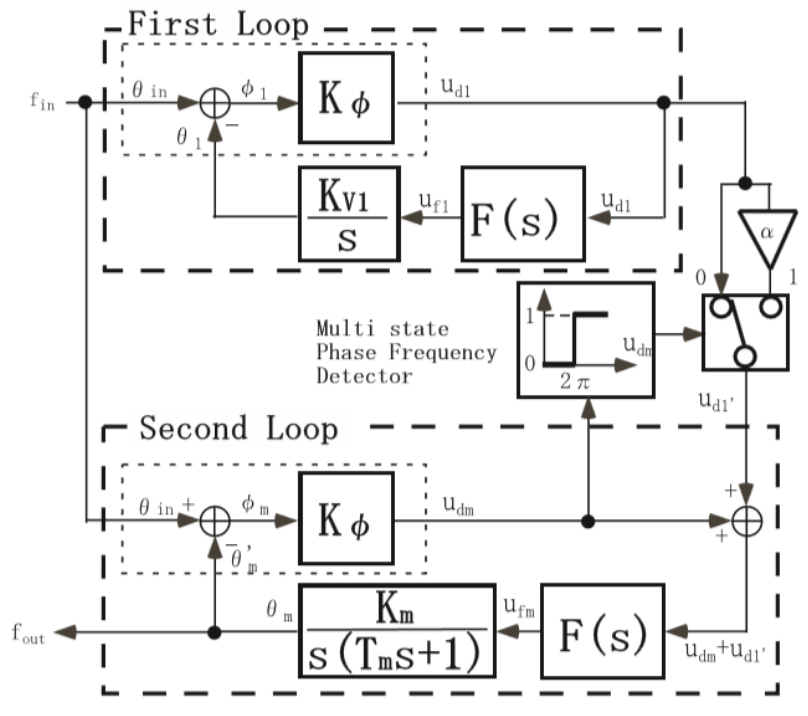

Fig. 7. proposed boost dual-loop PLL motor speed control system

\subsection{Simulation result}

In this subsection, a simulation result by Simulink is indicated in the Fig.8. When input signal is constant acceleration, a transition response of phase error by a conventional single-loop PLL-MSC is shown by a solid narrow line in Fig.8. In this case, large constant steady state phase error was occur. 


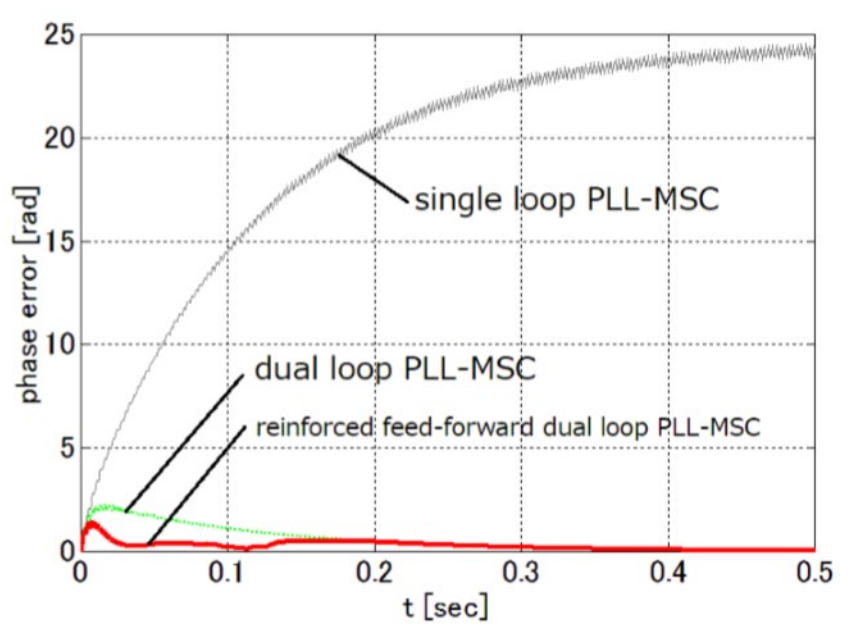

Fig. 8. phase error in the PLL-MSC

Therefore, a transition response of phase error by a dual-loop PLL-MSC is shown by dashed line in the Fig.8. In this case, phase error of the second loop is canceled by first loop ones.

Also, a transition response of phase error by a dual-loop PLL-MSC with reinforces feed-forward is shown by bold solid line of the Fig.8. In this case, phase error is limited by threshold level of the comparator for reinforcing.

However, there are discrete time and nonlinear behavior, so the analysis is very difficult. But, we are assuming this tuning method for the limited scale $\mathrm{XY}(\mathrm{Z})$ table of a machine tool, it can draw the best performance of the PLL-MSC.

\subsection{Experimental result}

Next, we show experimental result by prototype circuit shown in appendix to Fig.9, that is implementation by FPGA of the Fig. 11 circuit.

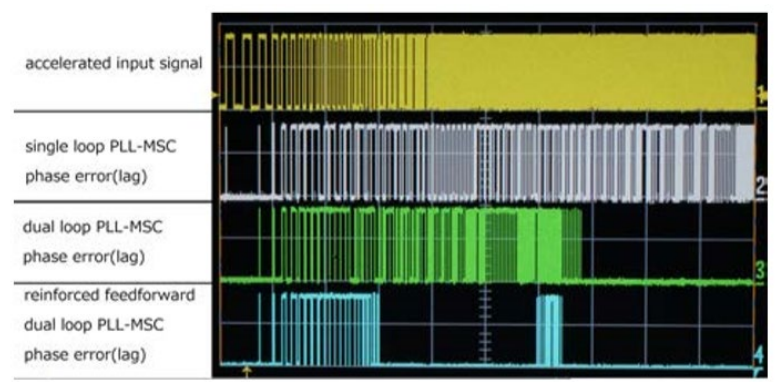

Fig. 9. experimental result of the various PLL motor speed control system

From top to bottom, first row is input in acceleratation, second is phase error in single-loop PLL-MSC, third is phase error in dual-loop PLL-MSC, and forth is phase error in the reinforced feed-forward dual-loop respectively.

It is clear, that random stripes are seen in the single-loop PLL-MSC which has a lot of cycle slip and harmful vibration, and all sometimes in the dual-loop PLL-MSC which has a little cycle slip, and the reinforced feed-forward dual-loop PLL-MSC has no cycle slip.

\section{X-Y table application}

Non-cycle slip PLL-MSC behaves just like stepping motor. In other word, there stepping motor can replace low-price DC brush motor.

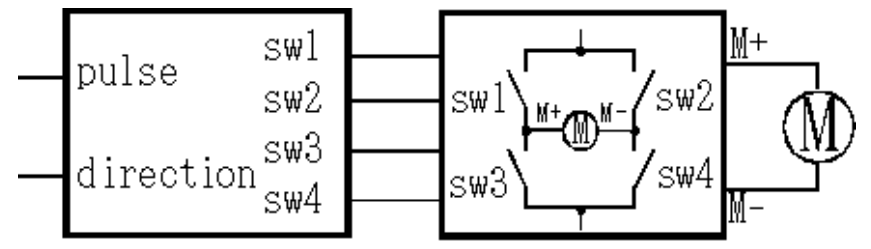

Fig.10 bi-direction DC brush motor driver

Therefore, we customize Fig.11 D.I.Y. mini CNC machine tool as experimental X-Y table that exchange from $\mathrm{X}, \mathrm{Y}$ stepping motor to $\mathrm{DC}$ motor with encoder.

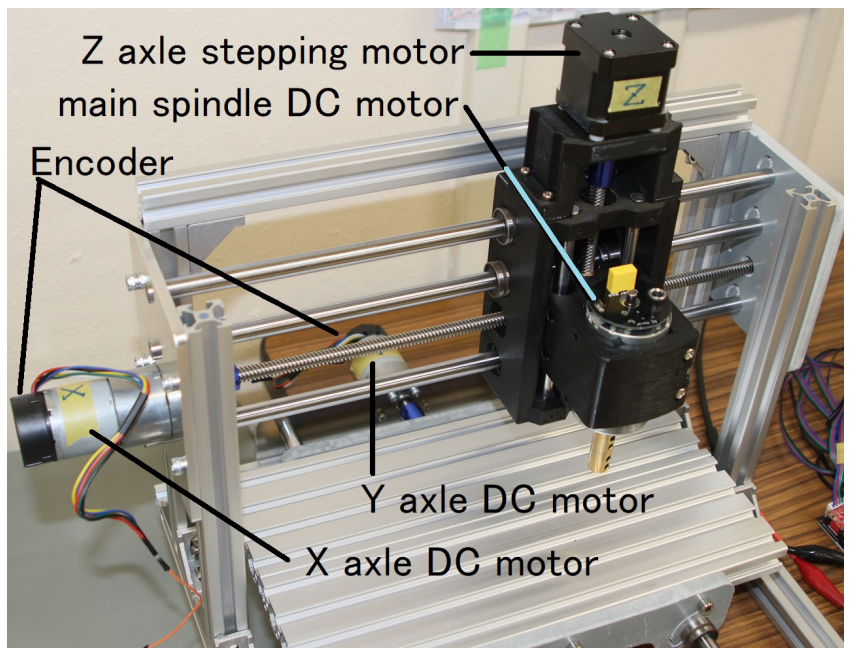

Fig.11 Experimental X-Y table is customized form mini

$\mathrm{CNC}$ machine tool

In this case, we use that Fig. 12 original stepping motor driver is outputting pulse train and the direction for PLL DC motor control input signal.

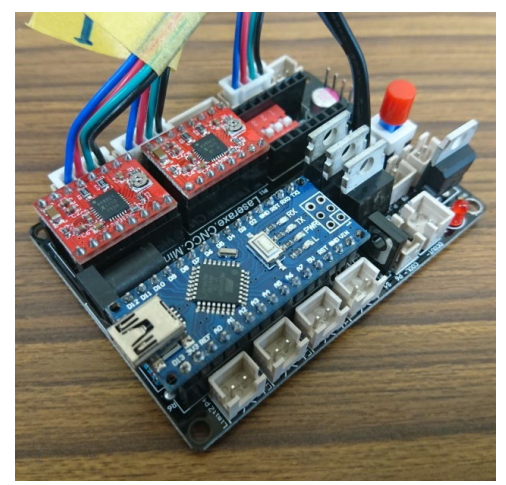

Fig.12 stepping motor driver is used as pulse generator. 
For these reference input signals, bi-direction DC brush motor driver Fig. 10 which has H-bridge, should be used.

We are planning to draw complete round using this experimental X-Y table.

\section{Conclusion}

In the PLL motor speed control system, input acceleration causes cycle slip. In this article, We propose prevention methods, by detecting multi-state PFD, the feed-forward amount is reinforced more aggressively in the dual-loop PLL-MSC. We are designing limited scale like the XY (Z) table of a machine tool, it can draw the best performance of the PLL-MSC.

\section{References}

(1) R.E.Best : "Phase-Locked Loops" 6th.ed.,McGraw-Hill, 2007

(2) A.W.Moore : "Phase-locked loop motor-speed control", IEEE Spectrum, pp.61-67, Apr. 1973

(3) H,Hosogaya : "Speed control of an industrial machine --- The applicability to the robot control by PLL control", 6,7th workshop of the Japan Society of Mechanical Engineers, 1986.

(4) M.Kamata, et al. : "Third-order phase locked loops using dual-loops inserting an active filter in the second loop with improved stability", IEICE-A, Vol.J82-A, No.2, pp.273-282, in Japanese, 1999

(5) H.Machida et al. : "Cycle slip prevention in PLL motion control using multi-state phase detector", ISCAIE 2018 in Pinag Malysia, 2018

Appendix Traditionally ${ }^{(3)}$, the PLL motor control including acceleration, detecting the slipped pulse and interpolating it to the input as shown in Fig.A. In this method, the precision couldn't be secured.

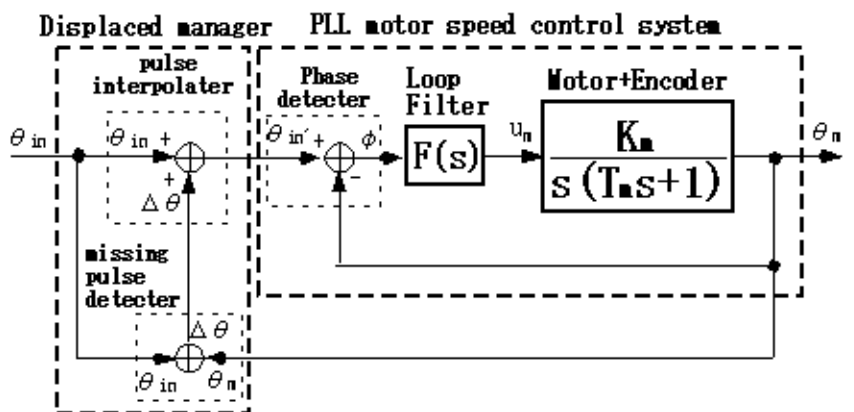

Fig.A Traditional PLL position control system 